\title{
Mientras haya vida, hay todo: una mirada en la sexualidad del adulto mayor
}

\author{
While there is life, there is everything: a look at the elderly sexuality
}

Eleonora Ghidara ${ }^{\mathrm{a}}$, Alberto Antacle $^{\mathrm{a}}$, Franco Erbetta ${ }^{\mathrm{a}}$, María Victoria Ferro ${ }^{\mathrm{a}}$, Melisa Fitzsimons $^{\mathrm{a}}$, Martín Loandos $^{\mathrm{a}}$, María Eugenia Luque $^{\mathrm{a}}$, María Ceclilia Melhen ${ }^{\mathrm{a}}$, Jessica Sal ${ }^{\mathrm{a}}$, Rosalía Sierra ${ }^{\mathrm{a}}$, Melissa Valdez Donelli ${ }^{\mathrm{a}}$, Emilio Verón $^{\mathrm{a}}$, María Julia Yanicelli ${ }^{\mathrm{a}}$

\begin{abstract}
Resumen
Introducción. Desde un punto de vista integral, la sexualidad comprende aspectos de orden biopsicosocial. Los prejuicios y el desconocimiento llevan a evitar hablar sobre este tema en la consulta con el adulto mayor (AM).

Objetivo. Profundizar en la vivencia de la sexualidad del AM.

Metodología. Estudio cualitativo, fenomenológico, realizado en dos centros de actividades recreativas para AM en San Miguel de Tucumán, que incluyó 13 entrevistas semiestructuradas en profundidad a pacientes mayores de 60 años, 11 de sexo femenino, seleccionados en forma intencional y por conveniencia en Agosto de 2018.

Resultados. Existe una dicotomía entre quienes hablan del tema y quienes no; sin embargo, todos expresan el deseo de naturalizarlo tanto en su círculo social como en la consulta médica. Los AM aceptan el proceso de envejecimiento, tienen una autopercepción positiva de su imagen y pese a los prejuicios, experiencias, comorbilidades y adversidades, se adaptan buscando formas para sentirse bien y disfrutar de una sexualidad plena.

Conclusión. El envejecimiento y la sexualidad son conceptos dinámicos que confluyen con las experiencias de vida. Creemos que como médicos de familia es importante brindar una atención integral, abordando las diferentes dimensiones del ser humano, incluyendo su sexualidad.
\end{abstract}

\begin{abstract}
Introduction. Sexuality from an integral point of view includes aspects of biopsychosocial order. Prejudice and ignorance lead to avoid talking about this topic in the medical consultation with the Eldery.

Objective. To deepen the experience of the sexuality of the Eldery.

Methodology. Qualitative, phenomenological study, conducted in two centres of recreational activities for Senior Citizens in San Miguel de Tucumán, which included 13 in-depth semi-structured interviews to patients over 60 years of age, 11 female, selected intentionally and for convenience in August 2018.

Results. There is a dichotomy between those who talk about the subject and those who do not; however, all of them express the desire to naturalize it in both, their social circle and the medical consultation. The eldery patients accept the ageing process, have a positive self-perception of their image and despite prejudices, experiences, comorbidities and adversities, they adapt looking for ways to feel good and enjoy a full sexuality.

Conclusion. Aging and sexuality are dynamic concepts that converge with life experiences. We believe that, as family doctors, it is important to provide comprehensive care, addressing the different dimensions of the human being, where sexuality is included.
\end{abstract}

Palabras clave: anciano, envejecimiento, medicina integral, sexualidad, análisis cualitativo. Keywords: aged, aging, integrative medicine, sexuality, qualitative analysis.

Ghidara E, Antacle A, Erbetta F, Ferro MV, Fitzsimons M, et al.. Mientras haya vida, hay todo: una mirada en la sexualidad del adulto mayor. Evid Actual Pract Ambul. 2019;22(1):e001105.

\section{Introducción}

Se define sexualidad desde un punto de vista integral, como una de las dimensiones fundamentales e inherentes a los seres humanos, dejando atrás enfoques limitados que la reducen al área de lo biológico. De esta forma, la sexualidad comprende aspectos de orden psicológico y social, incluyendo términos como el de género, identidad, personalidad, pensamientos, valores, afinidades y acompañamiento, lo que posibilita desarrollar al máximo el potencial de una persona. Es así como desde esta perspectiva, se puede decir que en toda conducta humana se da una expresión de la sexualidad ${ }^{1}$.

Argentina tiene un bajo índice de natalidad y una esperanza de vida cercana a los 75,7 años (OPS de 2011), lo que está conduciendo a que cada vez tengamos una población más envejecida ${ }^{2}$. En Argentina, un $63 \%$ de la población mayor de 60 años considera que es posible el enamoramiento en esta etapa de la vida y $80 \%$ de las personas de 60 a 74 años afirma mantener una vida sexual activa. Asimismo, un $50 \%$ opina que la vida sexual en una persona mayor es importante pero no tanto como cuando se es joven ${ }^{3}$.

El campo de la sexualidad en la tercera edad es un área difícil de comprender, debido a la falta de conocimiento respecto a la interacción envejecimiento-sexualidad ${ }^{4}$. En nuestra sociedad, los aspectos vinculados con la sexualidad han significado, hasta hace poco, un tabú que se incrementa al asociarlo con la edad, delimitado en gran medida por los aspectos culturales que favorecen la presunción de que la sexualidad en la tercera edad es inexistente ${ }^{1,5}$

Sin embargo, el deseo sexual puede mantenerse a pesar de los años, a no ser que sea alterado por los procesos fisiopatológicos asociados al envejecimiento ${ }^{6}$. Sin embargo, la edad, por si misma, no constituye un factor con entidad propia para afectar las relaciones sexuales y/o el placer asociado a esta ${ }^{7}$. Además, vale destacar que tienen menor incidencia las limitaciones asociadas con este cambio de la etapa vital, que los prejuicios y los estereotipos que favorecen el recelo de las personas mayores ante

a Obra Social del Personal de la Construcción. Construir salud., San Miguel de Tucumán, Argentina. eleonoraghidara@gmail.com 
la práctica sexual (p. ej. los medios difunden una imagen de la sexualidad asociada a la juventud y a la belleza, de la que quedan violentamente excluidas desde los simbólico las personas mayores), que pueden verse inhibidas en la búsqueda de relaciones sexuales y en la exploración de nuevos comportamientos placenteros $^{6}$.

Durante la vejez se busca amor, a la par que comprensión, afecto, compañía, solidaridad y también erotismo ${ }^{8}$. La necesidad afectiva es permanente a lo largo de la vida de todo ser humano y que está vinculada en gran medida con las experiencias vividas, con la autoestima de cada persona, con su grado de realización personal, y con sus necesidades de amor y aceptación. Cuando estás necesidades afectivas no pueden ser vivenciadas con plenitud se convierten en una fuente de malestar psicológico, capaz de afectar el crecimiento personal, la estabilidad familiar y la integración social.

Sin embargo, los prejuicios sociales y la ignorancia llevan a muchos profesionales de la salud, al propio individuo y a la familia a evitar el tema de la sexualidad en el anciano ${ }^{9}$, lo que conduce a que muchas de las insatisfacciones sexuales de nuestros pacientes no sean consultadas y, en consecuencia, no sean diagnosticadas ni tratadas correctamente ${ }^{10}$.

Por lo anteriormente mencionado consideramos fundamental conocer que factores influyen en la vivencia de la sexualidad de las personas adultas mayores (AM). Este conocimiento contribuirá a que los profesionales de la salud contemos con insumos que nos ayuden a mejorar el carácter integral de nuestra atención. El objetivo general de nuestro estudio fue explorar la vivencia de la sexualidad de las personas AM mientras que los objetivos específicos fueron caracterizar la influencia del entorno sociocultural sobre la sexualidad de las personas AM, describir sus experiencias y comprender la percepción de su propia imagen.

\section{Métodos}

Estudio cualitativo desde un abordaje fenomenológico. Esta metodología, en lugar de asumir que existe una realidad "objetiva" y "externa", igual para todas las personas, entiende que la esencia de dicha realidad depende del modo en que es vivida y percibida por el sujeto, en una forma única y propia para cada ser humano. Estas características convierten a la fenomenología en el método más apropiado para nuestro propósito de investigación. Se trabajó en dos escenarios:

Escenario EPAM: El EPAM (Educación Permanente para Adultos Mayores) es un programa de educación no formal dependiente de la Secretaría de Extensión Universitaria de la Universidad Nacional de Tucumán (UNT). Recibe a adultos de más de 50 años que participan en diversos talleres, que se agrupan en diferentes áreas temáticas (actividades corporales, ciencias naturales, expresión artística y musical, expresiones literarias y fantásticas, humanidades y ciencias sociales e idiomas). En general, sus alumnos poseen un alto grado de formación y un buen nivel intelectual.

Escenario PAMI: EI PAMI (Programa de Atención Medica Integral) creó un programa de actividades preventivas y recreativas que se desarrolla en la Ciudad de San Miguel de Tucumán, con objetivo de incrementar las oportunidades de salud de los afiliados en términos de autonomía funcional, participación en el control de sus problemas de salud, integración social y esparcimiento. El programa ofrece a sus afiliados realizar talleres y cursos gratuitos (costura, canto y taichí, etc.).

\section{Criterios de inclusión}

Hombres o mujeres, mayores de 60 años con buena función cognitiva que asistían a talleres del EPAM o PAMI.

\section{Universo y unidad de estudio}

La unidad de estudio estuvo conformada por 13 personas mayores de 60 años, 11 mujeres y dos varones, que asistieron a los talleres presentes en dos centros recreativos de San Miguel de Tucumán (EPAM y PAMI).

\section{Técnica de Muestreo}

La selección fue realizada de forma intencional y por conveniencia, a través de los coordinadores de los talleres, buscando aquellos participantes (informantes clave) que fueran capaces de brindar la mayor y mejor información posible.

\section{Técnica de recolección de datos}

El equipo investigador realizó entrevistas individuales en profundidad en las instituciones antes mencionadas, dentro de una habitación destinada para tal fin. Estas fueron fueron audiograbadas previa autorización de los/as participantes.

\section{Análisis de los datos}

El análisis fue realizado mediante el uso de la Teoría Fundamentada, que implica el análisis y la interpretación mediante la lectura y la relectura de los textos obtenidos de la desgrabación de las entrevistas, su desconstrucción mediante codificación abierta, y su posterior reconstrucción por codificación teórica, creando categorías descriptivas que se fueron relacionando y sintetizando hasta obtener la hipótesis heurística.

\section{Consideraciones éticas}

El equipo investigador solicitó a los participantes el consentimiento informado de manera oral, en el que se explicó en qué consistía la entrevista y cuál era el objetivo del trabajo de investigación. Se les aclaró los participantes que la información sería confidencial y anónima y que no se usaría para ningún otro propósito fuera de los de esta investigación. El protocolo fue aprobado por el comité de ética de la Universidad nacional de Tucumán.

\section{Resultados}

A continuación describimos las categorías descriptivas obtenidas a partir del análisis de las desgrabaciones de las entrevistas semiestructuradas:

\section{Espejito, espejito}

Con el correr de los años el cuerpo de los AM va cambiando, siguiendo el proceso natural de envejecimiento, deletéreo, progresivo, intrínseco, universal e irreversible.

A pesar de ello, confiesan no perder la vitalidad, buscando la manera de sentirse activos "... trato de hacer las cosas que me hacen bien, que me hacen sentir bien y me ayudan a mantener el cuerpo..." (Mujer de 74 años, EPAM)

Por una parte intentan optimizar la imagen personal, para sentirse mejor consigo mismos "...me arreglo principalmente para mí...", y por el otro, tratan de mostrar a la sociedad una actitud positiva hacia la vida “... y después para los que me quieran mirar..." (Mujer de 66 años, EPAM), reflejando que no hay límites de edad para la belleza y el cuidado del cuerpo. Llegaron a declarar incluso que, a pesar de todos los obstáculos que se presentan a esta edad "... yo me amo, me miro al espejo y me amo" (Mujer de 70 años, EPAM).

\section{Bailar pegados}

La sexualidad trasciende lo corporal y toman protagonismo las muestras de afecto, las demostraciones físicas, “... nos abrazamos, nos besamos...", actitudes y gestos que demuestran complicidad "...nos miramos y ya sabemos lo que estamos pensando..." (Mujer 70 años, EPAM) y compañerismo en situaciones de la vida cotidiana “... Ilamándolo por teléfono, preguntándole como 
está, si necesita algo..." (Mujer de 67 años, PAMI), lo que documenta la importancia de la esfera emocional.

Los AM buscan crear un escenario en el cual el envejecimiento no sea un impedimento para el encuentro con el otro "... salimos solos, viajamos..." "... ponemos música y bailamos..." (Mujer de 60 años, EPAM).

\section{Contigo aprendí}

Como parte inherente a la persona, la sexualidad está influenciada por la personalidad y la historia de vida de cada uno, configurándose el presente acorde a ellas.

Algunos refieren haber tenido experiencias dolorosas que los marcaron a fuego, y las asocian con el sufrimiento, declarando su negación a compartir su vida con otro: "... mi miedo es volver a tener un hombre, que se enferme y que vuelva a morir..." (Mujer de 65 años, PAMI), "... me divorcié y ahí termino mi relación con el sexo opuesto..." (Mujer de 74 años, EPAM).

Otros, a pesar de haber tenido experiencias de infidelidades, llegaron a la aceptación y la armonía consigo mismos y con la pareja. "... es el hombre de mi vida a pesar de todas las canalladas que a veces hacen los hombres..., yo sigo siendo fiel a él... "(Mujer de 67 años, PAMI).

En contraposición, hay quienes a pesar de los momentos difíciles lograron superar las crisis y viven su sexualidad gratamente: "... tuve una etapa de mucha angustia y en ese momento sentí la compañía de mi marido, él estaba conmigo. .. "(Mujer de 70 años, PAMI); llegando, hoy en día, a declararse conformes con ella... “...33 años no es fácil, me siento muy querida por él... ”"(Mujer de 60 años, EPAM).

\section{El juego de las palabras prohibidas}

Los AM configuran y validan su vivencia de la sexualidad influenciados por los prejuicios y los estereotipos propios, de la familia y de la sociedad.

Están quienes prefieren no hablar del tema "... uno siempre lo ha tomado como una cosa tan prohibida...", reflejo de lo que vivieron en sus familias, "... porque nuestros padres jamás nos hablaron de esas cosas...", y de la misma forma lo transmitieron a las suyas "... a mis hijos los crié con este tabú..." (Mujer de 65 años, PAMI).

A su vez, el prejuicio también puede encontrarse en los hijos y nietos "... ellos piensan así, que a nuestra edad ya no debemos hacer 'eso'. Que ya no tiene que existir..." (Mujer de 65 años, PAMI).

La limitación para compartir conversaciones sobre este tema, además, puede darse entre los propios amigos: “... mis compañeras se escandalizan cuando yo les digo algo, para mi es lo más natural..." (Mujer de 70 años, EPAM).

Sin embargo, están aquellos que se sienten más cómodos al hablar del tema con la familia: "con mis hijos sí hablamos, porque en mi casa somos así de hablar, bastante abiertos", así como también entre los pares: "... hablamos mucho con todos de la sexualidad..." (Mujer de 60 años, EPAM).

Los AM expresan el deseo de naturalizar el tema, "...es algo que lo tienen que hablar...", no solo en su círculo social, sino también en la consulta médica, espacio en el que, según refieren, los profesionales de la salud poseen las mismas barreras a la hora de abordar la sexualidad: "... los médicos también tienen esa vergüenza..." (Mujer de 70 años, PAMI).

\section{La ley del deseo}

Por lo general, en esta etapa de la vida el deseo se ve atenuado por factores como el estado físico y el psicológico, las enfermedades crónicas y sus tratamientos, las creencias sobre la sexualidad en la vejez, la actitud y la predisposición hacia ésta, y la relación de pareja. "...y yo creo que va disminuyendo..." (Mujer de 60 años, EPAM):"... no tengo ningún tipo de deseo sexual..." (Mujer de 66 años, PAMI);“... es diabético. .. y le disminuye mucho..." (Mujer de 70 años, PAMI);“... y será que así es el proceso de la vejez, que cuando no va más, no va más..." (Mujer de 65 años, PAMI).

Aunque en algunos casos el deseo está conservado "...yo tengo deseo con ella [...], ella no tiene deseo, porque tiene un problema de memoria [...]. Me quedo con el deseo nada más..." (Varón de 85 años, PAMI).

A pesar de eso, estas mismas razones los conducen a la búsqueda de otras formas para lograr mantener relaciones sexuales satisfactorias "... se puede solucionar con la pastilla celeste..." (Mujer de 70 años, PAMI), “... no sabes cómo sufro durante las relaciones... empecé con vaselina..." (Mujer de 66 años, EPAM), demostrando una actitud positiva en esta etapa de la vida.

\section{Amar o morir}

Los AM tienen una mirada integral de la sexualidad, que difiere de los prejuicios que tiene gran parte de la sociedad. Esta mirada no se encuentra condicionada únicamente por la influencia de la edad sobre el cuerpo, sino que involucra diferentes esferas como la afectividad, el deseo, la seducción, las experiencias y el entorno de cada persona. La genitalidad pasa a ser complementaria de un aspecto más profundo.

Los AM conciben la sexualidad de una manera holística, consideran que forma parte de la vida, y que la prioridad de lo físico sobre lo emocional se va invirtiendo desde la juventud hacia la vejez.

"... es comprensión, cariño, entendimiento. . . acompañamiento...todo" (Mujer de 67 años, PAMI); "... forma parte de nuestras vidas...” (Mujer de 62 años, PAMI); “... mientras haya vida. .. hay todo..." (Mujer de 65 años, PAMI); " . . . como nosotros cambiamos, nuestra relaciones también cambian. .." (Mujer de 65 años, EPAM);“. . la relación afectiva y sexual no es lo mismo que cuando sos joven..." (Mujer de 70 años, PAMI).

\section{Discusión y convergencia de datos}

La sexualidad en las personas AM ha sido una temática poco abordada, no solo de un punto de vista cultural e histórico, sino también en el campo más específico de la gerontología.

Al hablar de sexualidad, tendemos a asociarla con deseo y las prácticas sexuales, deduciendo que los AM no la viven de una manera satisfactoria, ya que sus capacidades físicas se encuentran disminuidas por el envejecimiento. Como mencionan Orozco y Rodríguez ${ }^{4}$, estos estereotipos son favorecedores de la inhibición del AM en la búsqueda de nuevas relaciones y comportamientos placenteros.

La influencia de las experiencias, los prejuicios, la afectividad y todas las esferas que rodean a la persona forman su propia concepción y por lo tanto su sexualidad, con su consecuente vivencia. Esto puede ser explicado desde la teoría de la psicología cognitiva, donde la idea de campo se basa en el principio de interdependencia que existe entre la persona y su entorno, planteándose que nuestra percepción y evaluación de los eventos es crucial. Es por eso que las creencias positivas o negativas, configuran las relaciones sociales.

Según Feingold y Mazzela ${ }^{11}$, los hombres tienden a sentirse satisfechos con su cuerpo a pesar de la edad, mientras que las mujeres tienden a afirmar lo contrario. Nuestros hallazgos no coinciden con los de este autor ya que nuestras entrevistadas tienden aceptar el proceso de envejecimiento de una manera natural y mantienen una autopercepción positiva, lo que les ayuda a vincularse con sus pares. Sin embargo, esto último podría quizás estar condicionado por algún tipo de sesgo de selección de la 
muestra, dado que las entrevistadas fueron invitadas a participar a partir de su concurrencia a dos instituciones que promueven la vinculación social y quizás sus discursos no son representativos del colectivo general de los AM.

\section{Conclusión}

Nuestros AM entrevistados manifestaron que los profesionales de la salud no suelen hacerles preguntas respecto de su sexualidad. Como equipo de salud consideramos que es importante brindar una atención integral, abordando las diferentes dimensiones que componen el ser humano, incluyendo a la sexualidad como parte esencial de la persona a lo largo de toda la vida.

Como lo documentaron los testimonios recogidos, la sexualidad se encuentra condicionada por lo físico, por lo emocional y por las experiencias vividas, así como también por el concepto que expresa la sociedad en la que se encuentran inmersos los adultos mayores.

Documentamos que el envejecimiento y la sexualidad son conceptos dinámicos que confluyen con las experiencias de vida, y que se definen y resignifican constantemente. Son conceptos inseparables y lo que ocurre en uno repercute sobre el otro.

Podemos concluir que, pese a los cambios propios de la edad, las comorbilidades y las adversidades que conlleva esta etapa, muchos AM se adaptan y tienen una gran vitalidad, buscando formas para sentirse bien y disfrutar de una sexualidad plena.

Conflicto de interés de los autores: Este trabajo fue autofinanciado por el grupo investigador, que declaran no tener conflictos de interés respecto de su temática.

\section{Referencias}

1. Fallas-Ureña H, Valverde-Cerros OA. Sexualidad y salud sexual reproductiva en la adolescencia. San José, Costa Rica; 2000. Módulo de capacitación para personal de salud. Guía metodológica. Departamento de Medicina Preventiva Caja Costarricense del Seguro Social. Available from: https://ccp.ucr.ac.cr/bvp/texto/21/reproductiva.htm.

2. El sistema de salud argentino y su trayectoria de largo plazo: logros alcanzados y desafíos futuros. 1st ed. Buenos Aires: Programa Naciones Unidas para el Desarrollo.; 2011. Available from: http://www.paho.org/arg/images/Gallery/publicaciones/El\%20sistema\%20de\%20salud\%20argentino\%20\%20pnud\%20ops\%20cepal.pdf?ua=1.

3. Encuesta Nacional sobre Calidad de Vida de Adultos Mayores 2012. Ciudad Autónoma de Buenos Aires: Instituto Nacional de Estadística y Censos; 2014.Available from: http://www.desarrollosocial.gob.ar/biblioteca/encuesta-sobre-calidad-de-vida-de-adultos-mayores-2012/.

4. Vidal LNE. Estudio de sexualidad en la tercera edad. . Valdivia, Chile; 2006. Available from: http://cybertesis.uach.cl/tesis/uach/2006/fme.18e/doc/ fme.18e.pdf.

5. Villar F. Desarrollo adulto y envejecimiento desde un punto de vista sociocontextual. In: Psicología Evolutiva y Psicología de la Educación. Barcelona; 2003. p. 754-863. Proyecto docente. Available from: http://www.ub.edu/dppsed/fvillar/principal/pdf/proyecto/cap 12 ciclovital.pdf

6. Orozco-Mares I, Rodriguez-Márquez DD. Prejuicios y actitudes hacia la sexualidad en la vejez. . Psicología y ciencia social. 2006;8(1):3-10. Available from: http://www.redalyc.org/pdf/314/31480101.pdf.

7. Esguerra-Velandia I. Sexualidad después de los 60 años. Avances de enfermería. 2007;25(2):124-140. Available from: https://revistas.unal.edu.co/ index.php/avenferm/article/view/12816.

8. Sapetti A. La sexualidad en el adulto mayor. Psicodebate. 2013;13:61-78. Available from: https://doi.org/10.18682/pd.v13i0.362.

9. Orte-Socias C, Vives-Barceló M, ydia Sánchez-Prieto. Amor y sexualidad en la vejez. La historia contada por sus protagonistas. In: Gazquez JJ, Pérez-Fuentes M, Molero MM, Mercader I, ABBarragán, editors. Investigación en salud y envejecimiento.. vol. II. Almería: ASUNIVEP; 2015. p. 127-133. Available from: 10.13140/RG.2.1.3698.0966.

10. Laurencio-Vallina SC, Jiménez-Betancourt E, Sánchez-Masó Y. Vivencias afectivas y factores condicionantes en adultos mayores sin relación de pareja. MEDISAN. 2017;21(1):102-102. Available from: http://scielo.sld.cu/scielo.php?script=sci_arttext\&pid=S1029-30192017000100012.

11. Feingold A, Mazzella R. Gender differences in body image are increasing. Psychological science. 1998;9(3):190-195. Available from: http://dx.doi. org/10.1111/1467-9280.00036. 